\title{
Fünf Jahre und drei Monate im Einsatz für die Weiterbildung
}

Urs Martin Lïtolf,

Präsident des

Weiterbildungsausschusses

für Medizinalberufe
Korrespondenz:

Prof. Dr. Urs Martin Lütolf

Universitätsspital Zürich

Klinik für Radio-Onkologie Medizinische Radiologie

CH-8091 Zürich
Das Zustandekommen der bilateralen Abkommen Schweiz-EU hat unter anderem die Personenfreizügigkeit und somit die Anerkennung von Berufskategorien zum Inhalt. Die gegenseitige Anerkennung von Facharzttiteln - Titeln, die in Zukunft an einer staatlichen Instanz zuteil seien - machte eine Verordnung im FMPG, dem Gesetz von 1877, notwendig. Auf den 1. Juni 2002 trat dieses Gesetz in Kraft. Was die Weiterbildung anbelangt, war selbstverständlich, dass das neue Medizinalberufegesetz die Bereiche Ausbildung und Weiterbildung beinhalten sollte. Bis zum Inkrafttreten des Medizinalberufegesetzes bedurfte es einer Zwischenlösung im Bereich Weiterbildung und eines entsprechenden Gremiums des Weiterbildungsausschusses. Der Bundesrat hat in dieses Gremium Vertreter aus der Sanitätsdirektorenkonferenz, aus dem Bundesamt für Sozialversicherung, dem BAG, dem Leitenden Ausschuss, fünf Ärztinnen und Ärzte aus Praxis, Spital und Lehre sowie zwei Ärztinnen und Ärzte aus Praxis und Lehre delegiert.

Am 1. Januar 2005 übernahm ich von Dr. René Salzberg die Leitung dieser Kommission. Als Vizepräsidentin amtierte Frau Dr. Kondo-Oesterreicher aus Genf.

\section{Das MedBG kommt nicht zur Zeit: Eine Akkreditierung wird notwendig}

Die Eidgenossenschaft übernahm die von der FMH und der Zahnärztegesellschaft (SSO) bestehende Weiterbildungsinfrastruktur und sprach eine globale Akkreditierung für drei Jahre aus; dies in der Überzeugung, dass das Medizinalberufegesetz in dieser Frist Gültigkeit erhalten würde.

Dem war nicht so. Das Gesetz schrieb eine Akkreditierung nach drei Jahren vor; eine Gesetzesänderung, um diese Frist zu strecken, war undenkbar, und so hatte der Weiterbildungsausschuss seine wohl grösste Aufgabe in seiner fünfjährigen Lebenszeit. Die Akkreditierung der 44 Facharzttitel der FMH und der 4 Fachzahnarzttitel der SSO war eine «Generalstabsübung», die die Fachkompetenz innerhalb des
Weiterbildungsausschusses voll beanspruchte. Diesem Akkreditierungsprozess waren grundlegende Diskussionen vorausgegangen, unter anderem weil gemäss dem Freizügigkeitsgesetz jeder Facharzttitel als solcher akkreditiert werden musste, die FMH aber mit einer Weiterbildungsordnung viele der zu akkreditierenden Punkte zentral und in guter Art und Weise abdeckte. Im weiteren war die Aufgabe des Weiterbildungsausschusses nicht, die Akkreditierung selbst vorzunehmen, sondern für das EDI beratend zu wirken. Das OAQ (Organ für Akkreditierung/Qualitätssicherung) wurde mit der technischen Durchführung beauftragt. Bei einem derart knappen Zeitrahmen versuchte der Weiterbildungsausschuss als Vermittler nicht jeden Prozess sequentiell zu durchlaufen, sondern an einem Tisch kritisch, aber speditiv zu einer Meinungsbildung $\mathrm{zu}$ kommen. Impulse aus diesen Diskussionen für Verbesserungen sind in beiden Trägerorganisationen (FMH und SSO) aufgenommen worden. Die Auflagen zur Akkreditierung wurden im Jahr 2007 dem Vorsteher des EDI zugestellt.

\section{Beratung der Trägerorganisationen zur Qualitätsverbesserung; ein weiter Aufgabenbereich des WA}

Die Erkenntnis, dass die Weiterbildung über Inhalte und nicht über Anzahl Jahre zu definieren sei, wurde vom Weiterbildungsausschuss diskutiert und den Trägerorganisationen und den einzelnen Facharzt- bzw. Fachzahnarztbereichen übermittelt. Die Verknüpfung mit standespolitischen Anliegen muss möglichst aufgehoben werden. Hier ist im Bereich der FMH während der Zeit des Weiterbildungsausschusses Wesentliches geschehen.

\section{Anerkennung ausländischer Facharzttitel}

In der «Lebensperiode» des Weiterbildungsausschusses sind über 3500 ausländische Fachärztinnen und Fachärzte im Rahmen der bilateralen Verträge zu ihrer Anerkennung gekommen. Fast 2500 dieser Anerkennungen betreffen Kolleginnen und Kollegen aus Deutschland, etwa 500 aus 
Frankreich, 300 aus Italien, bedeutend weniger aus anderen Staaten. Die Anerkennung muss ohne Überprüfung der Weiterbildungsumstände im Ursprungsland erfolgen. So kann das Ursprungsland ohne weiteres eine Weiterbildungsperiode, die kürzer ist als in der Schweiz, als Norm haben, kann ohne Fremdjahr und mit einfachen Evaluationen, die nie den Standard unserer Facharztprüfungen erreichen, auskommen. Hier könnte das Label FMH zusätzlich zu einem Facharzttitel wieder Gewicht erlangen, um den Patientinnen und Patienten solche Unterschiede zu veranschaulichen.

\section{Übergabe der Geschäfte an die Sektion Weiterbildung der MEBEKO}

Die Aufgaben der Sektion Weiterbildung der MEBEKO werden im grossen und ganzen dieselben bleiben, die der Weiterbildungsausschuss wahrgenommen hat:

- Beratung des Departements des Innern in Fragen der Weiterbildung;

- Stellungnahme zu den Akkreditierungsanträgen;

- Anerkennung der ausländischen Weiterbildungstitel;
- Vorschläge an die Trägerorganisationen der Weiterbildungsprogramme zur Erhöhung der Qualität der Weiterbildung.

In der neuen Sektion ist das ärztliche und zahnärztliche Know-how stark reduziert worden, dafür nehmen Veterinäre, Pharmazeuten und Chiropraktiker Einsitz in die Kommission.

\section{Abschliessende Betrachtung}

Während fünf Jahren und dreier Monate konnten in einer bewegten Zeit Erfahrungen im Rahmen des Auftrags der Kommission gesammelt und Impulse für die Weiterbildung an die Trägerorganisationen weitergegeben werden. Die respektable Qualität der Weiterbildungen in der Schweiz wird im Rahmen der Anerkennung verschiedener Modelle gleichgesetzt. Die Wertschätzung des in der Weiterbildung Geleisteten und die Suche nach einer Deklarierungsmöglichkeit für die hohe Qualität bleiben den Leistungserbringern (FMH, SSO und allen Weiterbildungsstätten) zu wünschen. 